\title{
Seasonal fluctuation in susceptibility to insecticides within natural populations of Drosophila melanogaster: empirical observations of fitness costs of insecticide resistance
}

\author{
Takahiro Miyo ${ }^{1}$, Sumio Akai ${ }^{2}$, and Yuzuru Oguma ${ }^{3 *}$ \\ ${ }^{1}$ Doctoral Program in Biological Sciences, University of Tsukuba, Ibaraki 305-8752, \\ ${ }^{2}$ Department of Food and Nutrition, Yamanashi Gakuin Junior College, Kofu, \\ Yamanashi 400-0805, and \\ ${ }^{3}$ Institute of Biological Sciences, University of Tsukuba, \\ Ibaraki 305-8752, Japan
}

(Received 5 December 1999, accepted 3 April 2000)

\begin{abstract}
To investigate genetic variation and seasonal fluctuation in susceptibility to insecticides, natural populations of Drosophila melanogaster were collected from Katsunuma in mid summer and late fall for two consecutive years. After isofemale lines of each population collected in each season had been established in a laboratory, the susceptibility of each line to five insecticides, including permethrin, malathion, prothiophos, fenitrothion, and DDT, was examined. Lines of each population exhibited the broad ranges of variation in susceptibility to all chemicals. Comparison between populations in different seasons indicated that genetic variation in susceptibility to organophosphates fluctuated in consistency with the population size, in which the susceptibility increased in fall. In addition, highly significant correlations were observed among responses to organophosphates, and the correlations also fluctuated with seasons. On the other hand, genetic variation in susceptibility to permethrin and DDT was less fluctuated. These results suggest that not only a common resistance factor for organophosphate resistance but also different resistance factor(s) for each insecticide could be involved within a natural population, and that the fluctuation observed in the susceptibility to organophosphates could be associated with fitness costs of organophosphate resistance factor(s).
\end{abstract}

\section{INTRODUCTION}

The development of insecticide resistance is an evolutionary process, in which insecticides act on genetic variation in susceptibility to insecticides as selection pressure. The rate of progress in insecticide resistance is dependent mainly on genetic variation within populations and selection intensity (Crow, 1957). Therefore, the development of insecticide resistance provides a practical model system for investigating evolutionary process, especially Darwinian deterministic process (Russell et al., 1990; Guillemaud et al., 1998).

What maintains genetic variation within populations and how selection acts on this variation have been discussed in evolutionary biology (Barton, 1990). In maintaining genetic variation within populations, negative genetic correlation between fitness components has been

Edited by Masa-Toshi Yamamoto

* Corresponding author. E-mail: oguma@biol.tsukuba.ac.jp considered to play an important role (Rose, 1982). Although the importance of negative genetic correlation in maintaining genetic variation has been recognized especially in theoretical population genetics, its empirical experimental evidence has been limited because of complex biochemical and physiological pathways (Barton, 1990). In the process of the development of insecticide resistance, negatively correlated responses to one insecticide have been observed: reversion to susceptibility after having been ceased from insecticidal selection.

Reduction in insecticide resistance after having been released from insecticide application has been sometimes observed (Crow, 1957). The substitution at a locus from a susceptible to a resistant allele could change biochemical and physiological processes involved in development (Clarke and McKenzie, 1987). Especially, the factors for insecticide resistance have been thought to have negative pleiotropic effects on fitness components, such as viability and fertility, since their frequencies in a population had usually been low before insecticides were applied (Crow, 
1957). Therefore, the development of insecticide resistance could be encountered by intense natural selection, and release from insecticide selection could result in reduction in resistance (Crow, 1957). However, this theory has been questioned mainly because empirical field observations of fitness costs of resistance factors have been quite limited (McKenzie et al., 1982; Chevillon et al., 1997). In addition, this fitness cost has not yet been made clear at the molecular level (Guillemaud et al., 1998). Chevillon et al. (1997) reported the field studies on fitness costs of organophosphate-resistance factors in Culex pipiens and indicated the variation in fitness costs among three resistance genes, from none to a severe disadvantage.

The goal of the present study is to reveal the genetic basis of negatively correlated responses to one insecticide. To do this, natural populations of $D$. melanogaster were first collected from Katsunuma in Japan in different seasons for two consecutive years. After isofemale lines of each population collected in different seasons had been established, the susceptibility of each isofemale line to each of five insecticides was evaluated. Since genetic variation within a natural population can be estimated from comparison among isofemale lines (Henter and Via, 1995; Imasheva et al., 1998), we investigated here how genetic variation in susceptibility to insecticides within a natural population fluctuated as population sizes changed with seasons and reported empirical field observations of the fitness cost(s) of resistance gene(s).

\section{MATERIALS AND METHODS}

Collection of natural populations of $D$. melanogaster and their rearing conditions. Natural populations of D. melanogaster at Katsunuma were collected in different seasons for two consecutive years (Table 1). Katsunuma is famous for its vineyards, and organophosphates have been mainly applied for controlling grape pests, including aphids, spider mites, and scales. We chose Katsunuma as a collection site because insecticides have been regularly applied to fields for pest control although Drosophila flies are not main targets.

Flies were captured, generally using several bananayeast bait traps placed at vineyards. Depending on the

Table 1. The number of Drosophila females collected in different seasons at Katsunuma

\begin{tabular}{lrrrrr}
\hline \hline Date & $\mathrm{n}$ & \multicolumn{2}{c}{ D. melanogaster ㅇ } & \multicolumn{2}{c}{ D. simulans ㅇ } \\
\hline Jul. 31, 1997 & 279 & 80 & $(0.287)$ & 199 & $(0.713)$ \\
Oct. 22, 1997 & 551 & 499 & $(0.906)$ & 52 & $(0.094)$ \\
\hline Jul. 31, 1998 & 1042 & 52 & $(0.050)$ & 990 & $(0.950)$ \\
Nov. 06, 1998 & 425 & 246 & $(0.579)$ & 179 & $(0.421)$ \\
\hline
\end{tabular}

Values within parentheses indicate the relative frequencies of females of each species in each season. abundance of flies, they were just swept or sucked up without baits. The $D$. melanogaster females collected were distinguished from $D$. simulans females, based on the observation of ovipositors under a microscope (Okada, 1956). Immediately after identification, 52 to 499 isofemale lines of each population were established in a laboratory. The flies used for experiments were grown on glucose-yeast-cornmeal-agar medium in incubators at 25 $\pm 0.5^{\circ} \mathrm{C}$ in a $14 \mathrm{~L}: 10 \mathrm{D}$ cycle. Otherwise flies were maintained on the same medium in an incubator at $18 \pm 0.5^{\circ} \mathrm{C}$ in a $14 \mathrm{~L}$ : $10 \mathrm{D}$ cycle.

Insecticides and bioassays. To evaluate the insecticide susceptibility of each isofemale line, five insecticides of a pyrethroid permethrin, organophosphates malathion, prothiophos, and fenitrothion, and an organochlorine DDT were chosen because these chemicals are typical of major classes of insecticides, and because some of them were or are indeed used at Katsunuma. Technical-grade permethrin (purity, 94.5\%), malathion (98.1\%), and fenitrothion (96.4\%) were obtained from Sumitomo Chemical Co., Ltd. (Osaka, Japan). Technical-grade prothiophos $(96.0 \%)$ was obtained from Nihon Bayer Agrochem K. K. (Tokyo, Japan). DDT (> 90\%) was kindly supplied by Dr. Shono at University of Tsukuba. Analytical-grade acetone (Wako Pure Chemical Industries, Ltd., Osaka, Japan) was used to dilute insecticides.

The filter-paper-contact method was used to evaluate the susceptibility of each line to each insecticide described above. Each insecticide was diluted with analyticalgrade acetone, and $100 \mu \mathrm{l}$ of the appropriate insecticide dilution was applied to a filter paper $(1 \times 8 \mathrm{~cm})$. One hundred microliters of acetone were applied as a control. After acetone had been evaporated, the filter paper was introduced into the glass vial $(2 \mathrm{~cm}$ diameter $\times 9 \mathrm{~cm}$ height) without food. Ten virgin adult females (4- to 6day old) were transferred into the vial, following a gentle ether anesthetization. These vials were then put into a plastic container $(14 \times 21 \times 17 \mathrm{~cm})$ with a paper towel, saturated with one milliliter of distilled water, and the containers were placed in incubators at $25 \pm 0.5^{\circ} \mathrm{C}$ in a 14 L: 10 D cycle. Mortality was scored after $24 \pm 0.5 \mathrm{~h}$, in which flies unable to walk normally were counted as dead.

Evaluation of the susceptibility of isofemale lines to insecticides. Based on the $\mathrm{LC}_{50}$ value of a standard laboratory line, Canton-S, the concentration of each insecticide for evaluating each isofemale line was determined (Table 2). The $\mathrm{LC}_{50}$ values of Canton-S were calculated from mortality data obtained for four or five concentrations of insecticides. The mortality data were pooled over replicates, corrected using Abbott's formula (Abbott, 1925), and then subjected to probit analysis (Proc Probit; SAS Institute, 1985).

Susceptibility of each isofemale line was evaluated as 
Table 2. Insecticide concentrations for evaluating each isofemale line

\begin{tabular}{lcc}
\hline \hline Insecticide & $\begin{array}{c}\text { Concentration } \\
\left(\mu \mathrm{g} / \mathrm{cm}^{2}\right)\end{array}$ & $\begin{array}{c}\text { Ratio to } \mathrm{LC}_{50} \\
\text { of Canton-S }\end{array}$ \\
\hline Permethrin & $1.25 \times 10^{3}$ & 3.0 \\
Malathion & 2.50 & 4.8 \\
Prothiophos & 0.313 & 3.9 \\
Fenitrothion & 0.625 & 17 \\
DDT & 125 & 49 \\
\hline
\end{tabular}

mortality under the determined concentration of each insecticide. Mortality data were pooled over replicates and corrected using Abbott's formula (Abbott, 1925). Arcsine-transformed mortality of each line was collected for each population in different seasons and subjected to analysis of variance (ANOVA) (Proc GLM; SAS Institute, 1985). Least significant difference (LSD) tests were conducted for comparisons of the mean arcsine-transformed mortality among populations $(P=0.05$; SAS Institute, 1985).

Correlations among responses to organophosphates. To evaluate how responses to insecticides were correlated and how these correlations fluctuated among seasons, the coefficients of determination were calculated after Pearson correlation coefficients among arcsine-transformed mortalities were obtained for each season (Proc CORR; SAS Institute, 1985).

\section{RESULTS}

Genetic variation in susceptibility to each insecticide within natural populations. In the mid-sum- mers of both 1997 and 1998, D. simulans was much more abundant than $D$. melanogaster at Katsunuma, in which more than 70 percent of females were $D$. simulans. However, D. melanogaster dominated $D$. simulans in the falls of both 1997 and 1998, in which the number of adult females of $D$. melanogaster collected was drastically increased (Table 1). Table 3 shows susceptibility to each of five insecticides within each population, where the mortality 0.000 and 1.000 is arcsine-transformed to 0.000 and 1.57, respectively. Although the number of isofemale lines established and tested was quite different among populations collected in different seasons, broad ranges of continuous genetic variation in susceptibility to each insecticide were observed among lines from each natural population collected in different seasons.

\section{Seasonal fluctuation in susceptibility to insecticides.} Natural populations of $D$. melanogaster at Katsunuma were collected four times for two years. The one-way ANOVA of the susceptibility to insecticides indicated a highly significant effect of collected seasons on the susceptibility to all chemicals, except for permethrin (Table 4).

For two consecutive years, seasonal fluctuation was observed in susceptibility to organophosphates (Table 3). Fig. 1 demonstrates the seasonal fluctuations in susceptibility to insecticides at Katsunuma. In cases of malathion and prothiophos, the mean mortality was significantly increased in the falls of 1997 and 1998, when the number of females of $D$. melanogaster collected were increased. In case of fenitrothion, like the other organophosphates, the mean mortality showed a tendency of an increased mortality in fall albeit without significance. On the other hand, susceptibility to permethrin and DDT was less fluctuated in different seasons in both 1997 and

Table 3. Comparison of susceptibility to five insecticides among populations collected in different seasons at Katsunuma

\begin{tabular}{|c|c|c|c|c|c|}
\hline \multirow{2}{*}{ Date } & \multicolumn{5}{|c|}{$\begin{array}{c}\text { Number of lines assayed } \\
\text { Mean arcsine-transformed mortality } \pm \text { SEM } \\
\text { (Minimum - Maximum) }\end{array}$} \\
\hline & Permethrin & Malathion & Prothiophos & Fenitrothion & DDT \\
\hline Jul. 31, 1997 & $\begin{array}{c}76 \\
0.627 \pm 0.0345^{\mathrm{a}} \\
(0.000-1.57)\end{array}$ & $\begin{array}{c}76 \\
0.505 \pm 0.0427^{\text {a }} \\
(0.000-1.57)\end{array}$ & $\begin{array}{c}76 \\
0.823 \pm 0.0421^{\mathrm{a}} \\
(0.000-1.57)\end{array}$ & $\begin{array}{c}76 \\
0.857 \pm 0.0442^{\mathrm{a}} \\
(0.183-1.57)\end{array}$ & $\begin{array}{l}76 \\
0.577 \pm 0.0322^{\mathrm{a}} \\
(0.000-1.39)\end{array}$ \\
\hline Oct. 22,1997 & $\begin{array}{c}260 \\
0.603 \pm 0.0151^{\mathrm{a}} \\
(0.000-1.20)\end{array}$ & $\begin{array}{l}286 \\
0.653 \pm 0.0214^{\text {bc }} \\
(0.000-1.57)\end{array}$ & $\begin{array}{l}260 \\
0.945 \pm 0.0262^{\mathrm{b}} \\
(0.000-1.57)\end{array}$ & $\begin{array}{l}283 \\
0.877 \pm 0.0218^{a} \\
(0.000-1.57)\end{array}$ & $\begin{array}{l}257 \\
0.532 \pm 0.0173^{\mathrm{ab}} \\
(0.000-1.57)\end{array}$ \\
\hline Jul. 31, 1998 & $\begin{array}{c}40 \\
0.574 \pm 0.0372^{\mathrm{a}} \\
(0.000-1.03)\end{array}$ & $\begin{array}{c}40 \\
0.576 \pm 0.0451^{\mathrm{ab}} \\
(0.000-1.15)\end{array}$ & $\begin{array}{c}40 \\
0.545 \pm 0.0397^{\mathrm{c}} \\
(0.000-1.11)\end{array}$ & $\begin{array}{c}40 \\
0.602 \pm 0.0395 \\
(0.183-1.25)\end{array}$ & $\begin{array}{c}40 \\
0.443 \pm 0.0319 \\
(0.000-0.886)\end{array}$ \\
\hline Nov. 06, 1998 & $\begin{array}{c}94 \\
0.667 \pm 0.02288^{\mathrm{a}} \\
(0.000-1.24)\end{array}$ & $\begin{array}{c}95 \\
0.742 \pm 0.0546^{\mathrm{c}} \\
(0.000-1.57)\end{array}$ & $\begin{array}{c}95 \\
0.765 \pm 0.0335^{\mathrm{a}} \\
(0.000-1.57)\end{array}$ & $\begin{array}{c}95 \\
0.659 \pm 0.0426^{b} \\
(0.000-1.57)\end{array}$ & $\begin{array}{c}94 \\
0.418 \pm 0.0334^{\mathrm{c}} \\
(0.000-1.57)\end{array}$ \\
\hline
\end{tabular}

Means with the same letter were not significantly different (LSD test, $P>0.05$ ). 
Table 4. One-way ANOVA for susceptibility to several insecticides among populations collected in different seasons at Katsunuma

\begin{tabular}{|c|c|c|c|c|c|c|c|c|c|c|c|c|c|c|c|}
\hline \multirow{2}{*}{$\begin{array}{l}\text { Source of } \\
\text { Variation }\end{array}$} & \multicolumn{3}{|c|}{ Permethrin } & \multicolumn{3}{|c|}{ Malathion } & \multicolumn{3}{|c|}{ Prothiophos } & \multicolumn{3}{|c|}{ Fenitrothion } & \multicolumn{3}{|c|}{ DDT } \\
\hline & d. f. & MS & $\mathrm{F}$ & d. f. & MS & $\mathrm{F}$ & d. f. & MS & $\mathrm{F}$ & d. f. & MS & $\mathrm{F}$ & d. f. & MS & $\mathrm{F}$ \\
\hline Populations & 3 & 0.121 & $1.96^{\mathrm{ns}}$ & 3 & 0.867 & $5.51^{* * *}$ & 3 & 2.27 & $15.3^{* * *}$ & 3 & 1.78 & $12.9 * * *$ & 3 & 0.483 & $6.04 * * *$ \\
\hline Error & 466 & 0.0617 & & 493 & 0.157 & & 467 & 0.148 & & 490 & 0.138 & & 463 & 0.0798 & \\
\hline
\end{tabular}

ns not significant, $* * * P<0.001$.

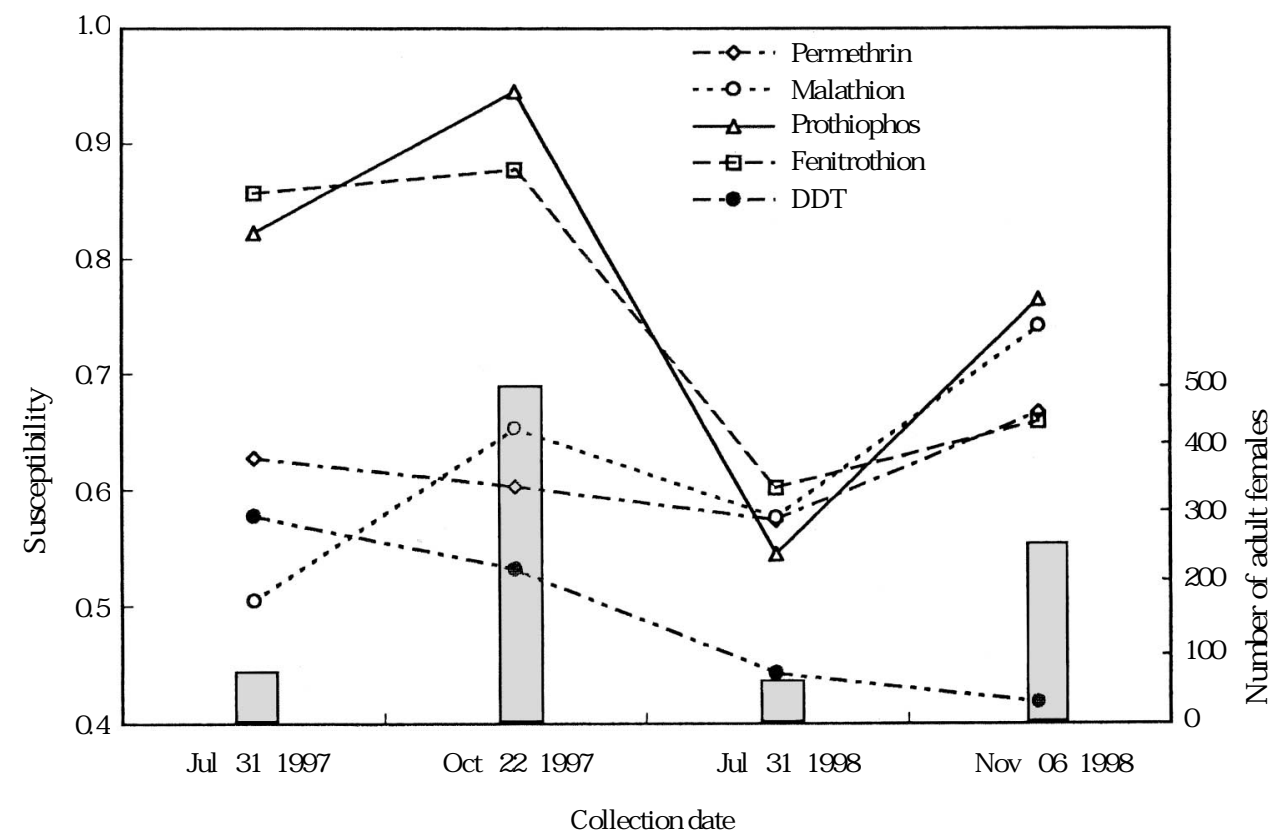

Fig. 1. Seasonal fluctuations in the number of adult females and susceptibility to insecticides at Katsunuma. Each line indicates susceptibility to each insecticide, expressed as mean arcsine-transformed mortality among isofemale lines. Shaded bar indicates the number of collected adult females of $D$. melanogaster.

Table 5. Correlations among responses to five insecticides and their fluctuation among seasons

\begin{tabular}{|c|c|c|c|c|c|}
\hline & & Permethrin & Malathion & Prothiophos & Fenitrothion \\
\hline \multirow{4}{*}{ Jul. 31, 1997} & Malathion & $0.376^{* * *}$ & & & \\
\hline & Prothiophos & $0.243^{*}$ & $0.584^{* * *}$ & & \\
\hline & Fenitrothion & $0.250^{*}$ & $0.716^{* * *}$ & $0.476^{* * *}$ & \\
\hline & DDT & $0.212^{\mathrm{ns}}$ & $0.076^{\mathrm{ns}}$ & $0.246^{*}$ & $0.260^{*}$ \\
\hline \multirow{4}{*}{ Oct. 22,1997} & Malathion & $0.059^{\mathrm{ns}}$ & & & \\
\hline & Prothiophos & $0.063^{\mathrm{ns}}$ & $0.357 * * *$ & & \\
\hline & Fenitrothion & $-0.041^{\mathrm{ns}}$ & $0.622^{* * *}$ & $0.335^{* * *}$ & \\
\hline & DDT & $0.212 * * *$ & $0.131^{*}$ & $0.189^{* *}$ & $0.175^{* *}$ \\
\hline \multirow{4}{*}{ Jul. 31, 1998} & Malathion & $-0.030^{\mathrm{ns}}$ & & & \\
\hline & Prothiophos & $-0.073^{\mathrm{ns}}$ & $0.531 * * *$ & & \\
\hline & Fenitrothion & $0.073^{\mathrm{ns}}$ & $0.632 * * *$ & $0.420 * *$ & \\
\hline & DDT & $0.442^{* *}$ & $-0.026^{\mathrm{ns}}$ & $0.174^{\mathrm{ns}}$ & $-0.109^{\mathrm{ns}}$ \\
\hline \multirow{4}{*}{ Nov. 06, 1998} & Malathion & $0.058^{\mathrm{ns}}$ & & & \\
\hline & Prothiophos & $0.160^{\mathrm{ns}}$ & $0.373^{* * * *}$ & & \\
\hline & Fenitrothion & $-0.009^{\mathrm{ns}}$ & $0.337 * * *$ & $0.340^{* * *}$ & \\
\hline & DDT & $0.056^{\mathrm{ns}}$ & $-0.008^{\mathrm{ns}}$ & $-0.126^{\mathrm{ns}}$ & $0.008^{\mathrm{ns}}$ \\
\hline
\end{tabular}

${ }^{\mathrm{ns}} P>0.05,{ }^{*} P \leqq .05, * * P<0.01, * * * P<0.001$. 


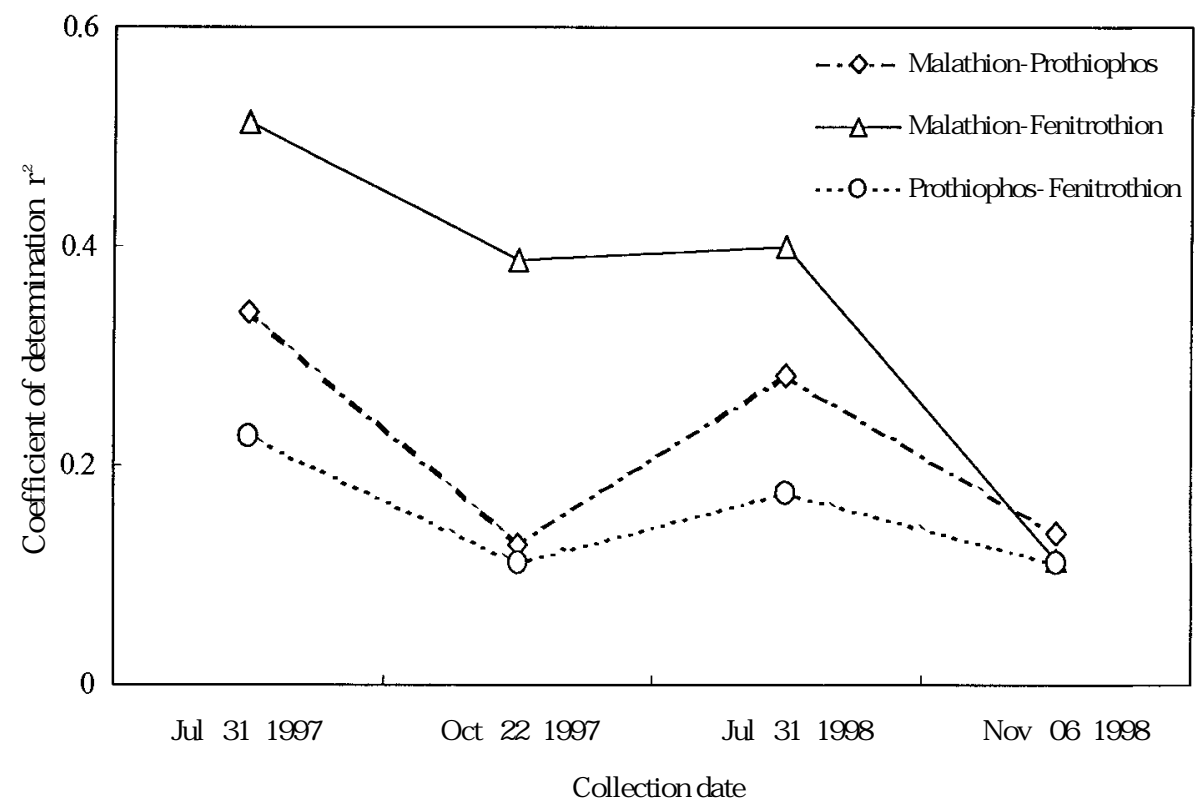

Fig. 2. Seasonal fluctuations in coefficients of determination. The coefficients of determination were obtained as the square values of correlation coefficients among responses to organophosphates.

1998. In July 1998, the mortality tended to be lower than that of 1997, which was observed for all chemicals (Table 3 and Fig. 1).

Correlations among responses to organophosphates and their fluctuation among seasons. Pearson correlation coefficients among responses to five insecticides were obtained for each season (Table 5). Although correlations among responses to organophosphates were highly significant for each season, correlations among responses to different classes of chemicals were not always significantly different from zero. Correlations among responses to organophosphates fluctuated with seasons, in which the coefficients of determination, the square values of correlation coefficients, tended to decrease in fall (Fig. 2). In cases of organophosphates, the coefficients of determination ranged from 0.127 to 0.341 between malathion and prothiophos, 0.114 to 0.513 between malathion and fenitrothion, and 0.112 to 0.227 between prothiophos and fenitrothion.

\section{DISCUSSION}

Genetic variation in insecticide resistance within natural populations. Broad ranges of continuous genetic variation in susceptibility to insecticides were observed in each population collected in different seasons (Table 3). Broad ranges of continuous genetic variation were also observed in natural populations collected at different locations (in preparation). These observations suggest that insecticide resistance could be a continuous quantitative trait at a population level.
The question of how many genes are responsible for insecticide resistance has been a controversial issue since 1950's, especially for DDT resistance in D. melanogaster (Dapkus, 1992). The genetic basis of insecticide resistance in $D$. melanogaster has not been made clear so far (Dapkus, 1992). Using a laboratory-selected cage population, of which a base population was a mixture of wild and laboratory stocks, Crow $(1954,1957)$ showed that DDT resistance factors were distributed to all major chromosomes. Using mixed populations of flies, Oshima (1954) obtained the similar results that both two major autosomes were involved in adult resistance to DDT, in which the second chromosome was more important. Dapkus and Merrell (1977) conducted chromosomal analysis of DDT resistance, indicating that all three major chromosomes contributed to DDT resistance although a small number of resistance genes might be involved. On the other hand, Ogaki and Tsukamoto (1953), Tsukamoto and Ogaki $(1953,1954)$ genetically analysed DDT resistance and located the major factor for larval resistance to DDT at about 66 on the second chromosome of $D$. melanogaster.

Raymond and Marquine (1994) studied resistance to an organophosphate, temephos, in a natural population of Culex pipiens and suggested that a relatively large number of resistance genes contributed to organophosphate resistance in that population, including an altered acetylcholinesterase and overproduced esterases. In addition, the examination of cross-resistance to two chemicals, chlorpyrifos and propoxur, indicated that one or some of resistance factors were responsible for cross-resistance. In this study, highly significant correlations among responses to organophosphates were observed in each sea- 
son (Table 5). In addition, fluctuation in correlations among responses to organophosphates exhibited the same tendency, in which coefficients of determination were decreased when the population size of $D$. melanogaster increased (Fig. 2). These observations suggest that there could exist a common factor responsible for resistance to organophosphates in this population. However, based on the coefficients of determination, less than half of variation in responses to one insecticide could be explained by variation in responses to another insecticide (Fig. 2). In other words, more than half of variation in responses to that insecticide should be explained by other factor(s). This could indicate that not only might a common factor responsible for organophosphate resistance contribute to resistance but other factor for resistance to each insecticide might be also involved in this population. Since broad ranges of continuous genetic variation in susceptibility to each insecticide were observed within the natural populations collected in different seasons (Table 3), several factors could contribute to insecticide resistance in natural populations.

Fluctuation in susceptibility to insecticides. It has been considered that genes responsible for insecticide resistance had fitness costs since the frequency of resistance genes in a population had been low before insecticides were applied (Crow, 1957; Dobzhansky et al., 1977). However, this hypothesis has been limited for its support from field observations (Roush and McKenzie, 1987). Chevillon et al. (1997) reported the results of the field studies on pleiotropic fitness costs of organophosphate-resistance genes in Culex pipiens. They showed the variation in fitness costs among different resistance genes.

Seasonal fluctuation in susceptibility to chemicals was observed in this study, in which susceptibility to organophosphates was increased in fall (Table 3 and Fig. 1). In addition, correlations among responses to organophosphates fluctuated, in which the coefficients of determination decreased in fall (Fig. 2). This could be a rare case in which reversion in insecticide resistance was observed at the level of genetic variation within a field population.

The population size of Drosophila in Japan is affected by many factors, including temperatures of the preceding winter (Watanabe et al., 1984) and other meteorological factors as well as temperatures (Mikasa, 1991). Concerning the Katsunuma population, it is well known that the seasonal distribution pattern of $D$. melanogaster is unimodal with a single peak in fall. Katsunuma is famous for its vineyards and wine production, and numbers of vineyards expand continuously at Katsunuma and surrounding areas. Until fall, D. melanogaster is considered to feed on dropped grapes. However, in fall, masses of squeezed grapes are created in the process of wine production and dumped outside. D. melanogaster flies feed and breed on the masses of squeezed and dumped grapes; as a result, the population size of $D$. melanogaster increases drastically in fall. In this study, the number of collected D. melanogaster increased drastically in fall, which could reflect the increase in the population size of D. melanogaster in fall (Table 1).

From spring until the vintage, pesticides, especially organophosphates, were applied to the fields for pest control. Although D. melanogaster is not a target of pesticides, insecticide selection pressure could be imposed indirectly on the fly population. In fact, the mean mortality was decreased in July 1998, compared with that in October 1997 (Table 3). However, flies could be released from insecticide selection in fall due to less application of insecticides since grapes had already been collected. As flies aggregated on the dumped grapes for feeding and breeding, the fly population at Katsunuma could grow drastically in fall (Fig. 1).

When a population size is far below the carrying capacity, the intrinsic rate of increase ( $r$ ) of each genotype determines the genetic composition of the population; that is, r-selection occurs (Roughgarden, 1971, 1996). In fall, Drosophila flies were released from insecticide selection, and resources for feeding and breeding (masses of squeezed grapes) increased suddenly and drastically at Katsunuma. As a result, $r$-selection against resistant individuals could occur there. Since a tendency to the increased mortality was consistent with the fluctuation in the population size (Fig. 1), this increased mortality could be associated with fitness disadvantage of genotypes resistant to organophosphates. Especially, since coefficients of determination decreased in fall (Fig. 2), the relative frequency of a common factor(s) responsible for organophosphate resistance might decrease in fall, due to fitness costs.

Migration of susceptible flies from surrounding areas could be another possible cause for this fluctuation. At this point, little has been revealed about the ecology and demography of $D$. melanogaster and $D$. simulans (Windelspecht et al., 1998). However, as mentioned earlier, numbers of vineyards expand continuously in Katsunuma and surrounding areas, and pesticides are applied to control grape pests. As a result, pesticide selection pressure could be imposed on the surrounding populations, as on the Katsunuma population. In fact, there were no significant differences in resistance levels between isofemale lines collected in the summer of 1997 at Katsunuma and at its surrounding area, Kofu (in preparation). In addition, we could not specify the reason why susceptible flies migrated into Katsunuma only in fall. Therefore, the increased population size and the increased susceptibility to insecticides in fall could not be due to migration of susceptible flies, but due to the difference in the intrinsic rate of growth between resistant and susceptible individuals within the Katsunuma population. 
Maintenance of genetic variation in susceptibility. It has been observed that a relatively large number of mutants resistant to insecticides existed in natural populations even though application of those insecticides had been terminated. Ffrench-Constant et al. (1990) isolated a dieldrin-resistant strain of $D$. melanogaster from natural populations, of which the resistance level was about 4,000 -folds. Since dieldrin usage had been terminated for about thirty years, it was suggested that dieldrin resistance was not associated with fitness disadvantages or that genetic variation in dieldrin resistance was maintained by other selection pressure, including other insecticides (Ffrench-Constant et al., 1990).

Resistant genotypes usually have lower fitness than susceptible genotypes under no insecticide conditions (Crow, 1957; McKenzie and Purvis, 1984). The insecticide application continued after development of the resistance in the population might cause the increased relative fitness of resistant genotypes even in insecticide-free environments (McKenzie and Purvis, 1984; Clarke and McKenzie, 1987). This compensation of fitness costs of resistance genes could be accomplished in two ways: the occurrence of modifier genes compensating fitness costs and replacement by less costly resistant genes (Guillemaud et al., 1998).

McKenzie et al. (1982) studied the effect of the genetic background on the fitness of genotypes resistant to an organophosphorus insecticide, diazinon, in Lucilia cuprina. They observed that there was no difference in fitness among the genotypes of diazinon resistance in a diazinonfree environment. However, if the genetic background was replaced by repeated backcrossing to a susceptible wild type, decreased fitness of resistant genotypes was observed; therefore, the genetic background modified the fitness of resistant genotypes (McKenzie and Purvis, 1984). In fact, the fitness modifier was mapped to the $w$ locus region of chromosome III, unlinked to the diazinon resistance locus on chromosome IV (McKenzie and Purvis, 1984; McKenzie and Game, 1987).

On the other hand, replacement by less costly resistant genes was also demonstrated. Guillemaud et al. (1998) studied evolution of organophosphate resistance due to overproduction of esterase in Culex pipiens in South France for 23 years. During this period, organophosphate insecticides were continuously applied to the fields for controlling C. pipiens (Guillemaud et al., 1998). They showed that the frequency of A1 esterase gene clinally distributed through an insecticide-treated and an insecticidenontreated area whereas that of A4-B4 esterase gene had a broad distribution, suggesting the difference in fitness cost among resistance genes. In addition, they showed that during this period, resistance genes were replaced from A1 to A4-B4, suggesting replacement of costly alleles by less costly alleles.

This study demonstrated the genetic variation in sus- ceptibility to DDT maintained in the Katsunuma population even a long period of time after termination of DDT application (Table 3). Since increased susceptibility to DDT was not observed in fall (Table 3) when the number of flies increased drastically, there could be no difference in fitness among the genotypes of DDT resistance. In addition, other selection pressure, rather than DDT itself, could be imposed on DDT-resistant genes since the susceptibility to DDT was gradually decreased through the collection periods (Fig. 1).

Since we have no information on the genetic constitution of the Katsunuma population during the period of DDT application, there is no way to specify the real causes of this maintenance. However, we have screened several isofemale lines exhibiting high levels of DDT resistance, and it could be possible to reveal the real causes by genetically analyzing these DDT-resistant lines.

Future prospects. In this study, it was suggested that the genotypes of organophosphate resistance in the Katsunuma population of D. melanogaster could have differences in fitness. At present, we are constructing chromosome-substituted resistant lines, of which backgrounds are substituted by that of a susceptible line. Genetic analyses using these chromosome-substituted lines could examine this hypothesis and, furthermore, reveal the genetic basis of negative correlations among insecticide resistance and fitness components.

We express our appreciation to Toshio Shono, Yoshiaki Kono, Kiyoshi Mizutani, Masahiro Ogaki and Sumiko Gamo for invaluable suggestions. We also thank Takeo Tadano for critical reading of the manuscript. We are grateful to Sumitomo Chemical Co. Ltd. and Nihon Bayer Agrochem K. K. for the kind supply of technical-grade insecticides.

\section{REFERENCES}

Abbott, W. S. (1925) A method of computing the effectiveness of an insecticide. J. Econ. Entomol. 18, 265-267.

Barton, N. H. (1990) Pleiotropic models of quantitative variation. Genetics 124, 773-782.

Chevillon, C., Bourguet, D., Rousset, F., Pasteur, N., and Raymond, M. (1997) Pleiotropy of adaptive changes in populations: comparisons among insecticide resistance genes in Culex pipiens. Genet. Res. Camb. 70, 195-204.

Clarke, G. M., and McKenzie, J. A. (1987) Developmental stability of insecticide resistant phenotypes in blowfly; a result of canalizing natural selection. Nature 325, 345-346.

Crow, J. F. (1954) Analysis of a DDT-resistant strain of Drosophila. J. Econ. Entomol. 47, 393-398.

Crow, J. F. (1957) Genetics of insect resistance to chemicals. Annu. Rev. Entomol. 2, 227-246.

Dapkus, D. (1992) Genetic localization of DDT resistance in Drosophila melanogaster (Diptera: Drosophilidae). J. Econ. Entomol. 85, 340-347.

Dapkus, D., and Merrell, D. J. (1977) Chromosomal analysis of DDT-resistance in a long-term selected population of Drosophila melanogaster. Genetics 87, 685-697. 
Dobzhansky, T., Ayala, F. J., Stebbins, G. L., and Valentine, J. W. (1977) Evolution. Freeman, San Francisco.

Ffrench-Constant, R. H., Roush, R. T., Mortlock, D., and Dively, G. P. (1990) Isolation of dieldrin resistance from field populations of Drosophila melanogaster (Diptera: Drosophilidae). J. Econ. Entomol. 83, 1733-1737.

Guillemaud, T., Lenormand, T., Bourguet, D., Chevillon, C., Pasteur, N., and Raymond, M. (1998) Evolution of resistance in Culex pipiens: allele replacement and changing environment. Evolution 52, 443-453.

Henter, H. J., and Via, S. (1995) The potential for coevolution in a host-parasitoid system. I. Genetic variation within an aphid population in susceptibility to a parasitic wasp. Evolution 49, 427-438.

Imasheva, A. G., Loeschcke, V., Zhivotovsky, L. A., and Lazebny, O. E. (1998) Stress temperatures and quantitative variation in Drosophila melanogaster. Heredity 81, 246-253.

McKenzie, J. A., and Game, A. Y. (1987) Diazinon resistance in Lucilia cuprina; mapping of a fitness modifier. Heredity $\mathbf{5 9}$, 371-381.

McKenzie, J. A., and Purvis, A. (1984) Chromosomal localisation of fitness modifiers of diazinon resistance genotypes of Lucilia cuprina. Heredity 53, 625-634.

McKenzie, J. A., Whitten, M. J., and Adena, M. A. (1982) The effect of genetic background on the fitness of diazinon resistance genotypes of the Australian sheep blowfly, Lucilia cuprina. Heredity 49, 1-9.

Mikasa, K. (1991) Statistical analysis of seasonal fluctuations in Drosophila populations near human habitation in Himeji City. J. Arts Sci. Meikai Univ. 3, 8-18.

Ogaki, M., and Tsukamoto, M. (1953) Genetical analysis of DDT-resistance in some Japanese strains of Drosophila melanogaster. Botyu-Kagaku 18, 100-104.

Okada, T. (1956) Systematic study of Drosophilidae and allied families of Japan. Gihodo, Tokyo.

Oshima, C. (1954) Genetical studies on DDT-resistance in popu- lations of Drosophila melanogaster. (1). Botyu-Kagaku 19, 93100.

Raymond, M., and Marquine, M. (1994) Evolution of insecticide resistance in Culex pipiens populations: the Corsican paradox. J. Evol. Biol. 7, 315-337.

Rose, M. R. (1982) Antagonistic pleiotropy, dominance, and genetic variation. Heredity 48, 63-78.

Roughgarden, J. (1971) Density-dependent natural selection. Ecology 52, 453-468.

Roughgarden, J. (1996) Theory of Population Genetics and Evolutionary Ecology: An Introduction. Prentice-Hall, New Jersey.

Roush, R. T., and McKenzie, J. A. (1987) Ecological genetics of insecticide and acaricide resistance. Annu. Rev. Entomol. 32, 361-380.

Russell, R. J., Dumancic, M. M., Foster, G. G., Weller, G. L., Healy, M. J., and Oakeshott, J. G. (1990) Insecticide resistance as a model system for studying molecular evolution. In: Ecological and Evolutionary Genetics of Drosophila (eds.: J. S. F. Barker, W. T. Starmer and R. J. MacIntyre), pp. 293-314. Plenum Press, New York.

SAS Institute (1985) SAS user's guide: statistics. SAS Institute, Cary, N. C.

Tsukamoto, M., and Ogaki, M. (1953) Inheritance of resistance to DDT in Drosophila melanogaster. Botyu-Kagaku 18, 39-44.

Tsukamoto, M., and Ogaki, M. (1954) Gene analysis of resistance to DDT and BHC in Drosophila melanogaster. BotyuKagaku 19, 25-32.

Watanabe, T. K., Inoue, Y., and Watada, M. (1984) Adaptation of Drosophila simulans in Japan. Jpn. J. Genet. 59, 225-235.

Windelspecht, M., Richmond, R. C., and Cochrane, B. J. (1998) Survey of malathion resistance and avermectin susceptibility in field populations of Drosophila melanogaster (Diptera: Drosophilidae) and D. simulans. J. Econ. Entomol. 91: 1245-1252. 